\title{
New Crossover Network Based on q-Bernstein Polynomial
}

\author{
Dolchai Sookcharoenphol ${ }^{1+}$, Hideyuki Nomura ${ }^{2}$ Chisato Kanamori ${ }^{3}$, Hisayuki Aoyama ${ }^{3}$, Kanok \\ Janjitrapongvej ${ }^{4}$ and Vanvisa Chutchavong ${ }^{1}$ \\ ${ }^{1}$ Faculty of Engineering, King Mongkut's Institute of Technology Ladkrabang, Bangkok, Thailand \\ ${ }^{2}$ Department of Communication Engineering and Informatics, University of Electro-Communications, \\ Tokyo, Japan \\ ${ }^{3}$ Department of Mechanical Engineering and Intelligent Systems, Graduate School of Informatics and \\ Engineering, University of Electro-Communications, Tokyo, Japan \\ ${ }^{4}$ Faculty of Science and Technology, Southeast Bangkok College, Bangkok, Thailand
}

\begin{abstract}
This paper presents a new audio crossover network based on q-Bernstein polynomial which can be adjustable attenuation in stop-band by varying parameters of the transfer function. The crossover network gives an in-phase response and a flat summed of magnitude response of each filter through audio frequency band. The simulation results shown that the crossover network given high attenuation in stop-band which better than conventional crossover network at second-order and fourth-order. Moreover, the crossover network structure can be reduced complexity by based on subtractive circuits. Therefore, the proposed crossover network provided a high quality crossover network and can useful for professional reproduction sound systems.
\end{abstract}

Keywords: q-Bernstein filter, in-phase crossover network, all-pole filter.

\section{Introduction}

Generally, a crossover network used to divide wide frequency of an audio band for suitable of operating driver frequency. A two-band crossover network contains a low-pass filter and a high-pass filter for divide low frequency signal apply to low frequency driver or called woofer and the other high frequency signal apply to high frequency driver or Tweeter. When a driver is add between high and low frequency band, it is called "midrange" which use band-pass filter(BPF) to filter only specific frequency band and the crossover network is named a three way crossover network.

In conventional crossover network can be realized both of passive or active network type. For high quality audio requirement obtained, the network should be follow below properties [1-4].

1. Summation of frequency response should flat (Flat magnitude response)

2. High slope

3. In phase for crossover frequency

4. Linear Phase response or group delay should be flat in pass band

A typical crossover network implementation, a fourth order Linkwitz-Riley [5] or LR4 filter is often used because it given 1 and 3 properties but it does not met 2 and 4 properties. In an audio acoustic engineering term, the third property is an important property of the system to related directing of sound radiation. However, if there are available networks that miss this property which caused the main beam of polar radiation pattern tilted on axis and sound quality perceived can be degraded [5], [7].

\footnotetext{
${ }^{+}$Corresponding author.

E-mail address: ksdolchai@gmail.com
} 
This paper presents a new crossover network which can be adjustable attenuation in stop band by changing $\alpha, q$ and epsilon parameters of the transfer function of q-Bernstein filter. In additional, the in-phase and flat magnitude characteristics is provided by the network over audio frequency band.

\section{Crossover Network}

\section{1. q-Bernstein filter}

In conventional designed filter used approximating low-pass filter by classical polynomials such as Butterworth polynomial, etc. It has only one parameter to control the stop band attenuation of the filter by change the order of the transfer function. Thus, it cannot provide variable parameter of the transfer function for change the frequency characteristics to meet the desired magnitude response, when the order is given. Therefore, the crossover network that implemented by such filters cannot meet a requirement of the high quality needed and affects to generating distortion of the output signals. According to [6], Bernstein polynomial is applied to be a video equalizer due to it gives a better characteristic of frequency responses. A Bernstein filter can be approximated by Bernstein polynomial which corresponding to the desired function $f(x)$ on the range of $(0-1)$. Therefore, the Bernstein polynomial order-n is written as follows

$$
B_{n}(f ; x)=\sum_{k=0}^{n} f\left(\frac{k}{n}\right) \cdot\left(\begin{array}{l}
n \\
k
\end{array}\right) x^{k} \cdot(1-x)^{n-k}
$$

When $\mathrm{f}(\mathrm{x})$ is a desired function to approximates and given that

$$
\cos ^{2} x+\sin ^{2} x=1
$$

From quantum calculus in reference [7] to instead $f(x)$ into $(1-a)^{n}$ form, we can rewriting as

$$
(1-a)_{q}^{n}=\prod_{j=0}^{n-1}\left(1-q^{j} \cdot a\right)
$$

Thus, from (1) and (2) when substituting $a=\left(\frac{y}{\alpha}\right)^{2}$ in (1), yields

$$
B_{n, q}(f ; x)=\sum_{k=0}^{n} f\left(\frac{k}{n}\right) \cdot\left(\begin{array}{l}
n \\
k
\end{array}\right)_{q} x^{k} \cdot(1-x)_{q}^{n-k}
$$

And also substituting (3) into (4) and rewritten by

$$
B_{n, q}\left(f ; y^{2}\right)=\sum_{k=0}^{n}\left(1-\left(\frac{k}{\alpha \cdot n}\right)^{2}\right)^{-1} \cdot\left(\begin{array}{l}
n \\
k
\end{array}\right) y_{q} y^{2 \cdot k} \cdot\left(1-y^{2}\right)_{q}^{n-k}
$$

An equation (5) is a general form of q-Bernstein polynomial of order $n$

And the relation of approximating functions and desired functions are

$$
\begin{aligned}
& f\left(\frac{k}{n}\right)=\left(1-\left(\frac{k}{\alpha . n}\right)^{2}\right)^{-1}=\cos ^{2} x ; \text { for LPF } \\
& f\left(\frac{k}{n}\right)=\left(1-\left(\frac{k}{\alpha \cdot n}\right)^{2}\right)^{-1}=\sin ^{2} x ; \text { for HPF }
\end{aligned}
$$

To obtain an approximating low-pass filter by using q-Bernstein polynomial, we can substituting (5) and (6) into (7), that is

$$
\left|H_{n, q}\left(f ; y^{2}\right)\right|^{2}=\frac{A_{o}^{2}}{\left[1+\varepsilon^{2} \cdot B_{n, q^{2}}\left(f ; y^{2}\right)\right]}
$$

We can changes a variable that lies in limited range to a new range, we can substituting an old variable $y$ to a new variable, yields $y^{2}=\left(\frac{\Omega^{2}}{1+\Omega^{2}}\right)$, for LPF and $y^{2}=\left(\frac{1}{1+\Omega^{2}}\right)$, for HPF. And to obtains a transfer function in s-domain, we can substituting $\Omega$ with as $\Omega=-j / s$.Thus from (7), the magnitude squared transfer function can rewriting in a rational polynomial form as

$$
H_{L}^{2}(s)=\frac{N_{n}^{2}(s)}{D_{n}^{2}(s)}
$$


When we is given an order of the system, i.e. $\mathrm{n}=2$, we can rewriting a numerator polynomial as

$$
N_{n}^{2}(s)=\left(1-s^{2}\right)^{2}
$$

And a denominator polynomial can write as

$$
D_{n}^{2}(s)=\left[s^{4}\left(1+\varepsilon^{2}(A+B)\right)-s^{2}\left(\varepsilon^{2} \cdot B+2\right)+(C+1)\right.
$$

where $A=q+\frac{\alpha^{2}}{\alpha^{2}-1}-(1-q) \cdot\left(\frac{4 \alpha^{2}}{4 \alpha^{2}-1}\right), B=(1+q)\left(\frac{4 \alpha^{2}}{4 \alpha^{2}-1}\right)-(q+1)$ and $\mathrm{C}=1$ are the coefficient of filter, respectively.

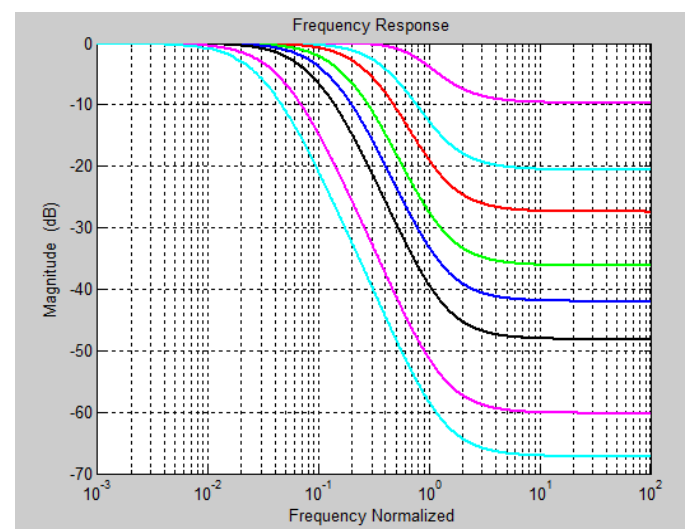

Fig. 1: Magnitude responses of $\mathrm{q}-B e r n s t a i n$ filter with varies stopband attenuation at $\alpha=0.55, \mathrm{q}=0.9$ and $\varepsilon=[0.51 .0$ $1.52 .53 .55 .01015]$.

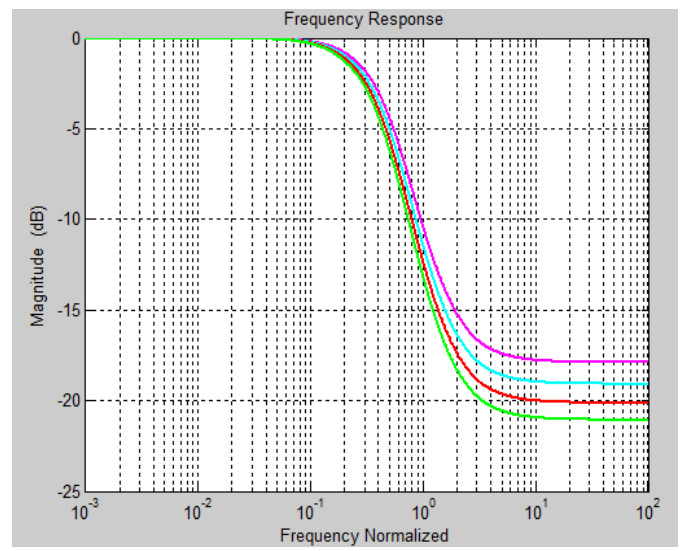

Fig. 2: Magnitude responses of q-Bernstain filter with varies stopband attenuation at $0.4<\mathrm{q}<1$, fixed $\varepsilon=1$ and $\propto=0.55$.

The magnitude of the q-Bernstein Low-pass filter with various parameters is shown as Fig.1. The stopband attenuation of the filter can be changed from $10 \mathrm{~dB}(\varepsilon=0.5)$ to $68 \mathrm{~dB} \varepsilon=15)$ by varies 3 parameter as

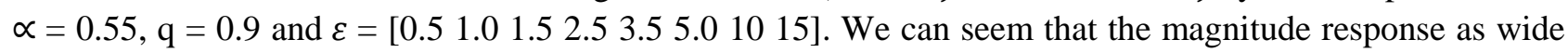
range changed or coase fine adjustment by $\varepsilon$ parameter. Whereas the magnitude is presented in Fig. 2 shown a fine adjustment by changed q parameter in range 0.4 to 1 with $0.2 \mathrm{~dB} / \mathrm{step}$ and another parameters are fixed as $\varepsilon=1$ and $\propto=0.55$. The magnitude in stop-band can change in narrow range of $\mathrm{dB}$. The magnitude response of the proposed filter is rather flat in pass-band which is one significant characteristic of the high quality filter for delicate audio field.

\subsection{Subtractive Structure}

A Conventional two-way crossover network consists of a low pass filter (LPF) and a high pass filter (HPF) is shown in Fig. 3 when gives H1(s) and H2(s) as LPF and HPF (without subtractive path), respectively. Generally, The Butterworth filter is often useful to implements of crossover network because it provides only a maximally flat magnitude response but all requirement properties cannot meet. A 
disadvantage of the direct structure is not guarantee to meet the requirement 1 , when used a second-order; and also complexness of the circuit is given. To reduction complexity, we can instead the $\mathrm{H} 2$ (s) with delayed process or all-pass filter (APF) [2] and [4]. Therefore, the output of the high frequency signal $\mathrm{Yu}(\mathrm{t}) \mathrm{can}$ be obtained by subtracting the output signal of the LPF and APF. The transfer function of the crossover network can be written in s-domain as

$$
H_{L P}(s)+H_{H P}(s)=A(s)
$$

where $H_{L P}(s)$ and $H_{L P}(s)$ are Low-pass and High-pass filters, respectively.

\subsection{In-Phase All-Pass Crossover Network}

Given that an stable all-pass filter defined as

$$
A\left(s_{n}\right) \triangleq \frac{D\left(-s_{n}\right)}{D\left(s_{n}\right)}
$$

where $\mathrm{D}\left(\mathrm{s}_{\mathrm{n}}\right)$ is a polynomial of stable system, i.e. Butterworth polynomials

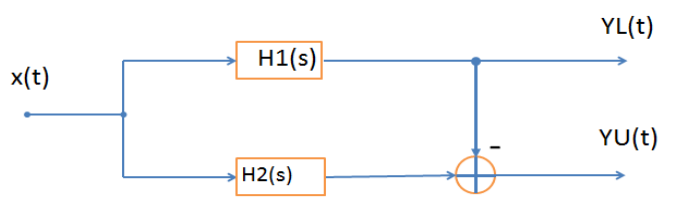

Fig. 3: Subtractive structure of a two band crossover network.

The LPF transfer function can be written in an all-pole low-pass filter form as

$$
\mathrm{H}_{\mathrm{L}}\left(\mathrm{s}_{\mathrm{n}}\right)=\frac{1}{\left[\mathrm{D}_{\mathrm{r}}\left(\mathrm{s}_{\mathrm{n}}\right)\right]^{2}}
$$

Since, from (13) the transfer function of the HPF is achieved by subtraction the output of the APF with the output of the LPF, yields

$$
\begin{gathered}
H_{H P}\left(s_{n}\right) \triangleq A\left(s_{n}\right)-H_{L P}\left(s_{n}\right) \\
=\frac{D\left(s_{n}\right) D\left(-s_{n}\right)-1}{\left[D\left(s_{n}\right)\right]^{2}}
\end{gathered}
$$

When substituting $s=j . \omega$ into (12)-(14), the magnitude response of these filter are obtained. Simulation Results of the proposed crossover network shows next section both two-band and three- band networks. For a three-band network, the transfer function of a three filters can be implemented by cascading of an APF transfer function and a constant voltage transfer function which are in second-order [8]. The frequency responses of these crossover networks are shows in next section.

\section{Simulation Results}

The magnitude and phase frequency responses of the two and three way crossover networks are shown in Fig. 4 and Fig. 5, respectively.

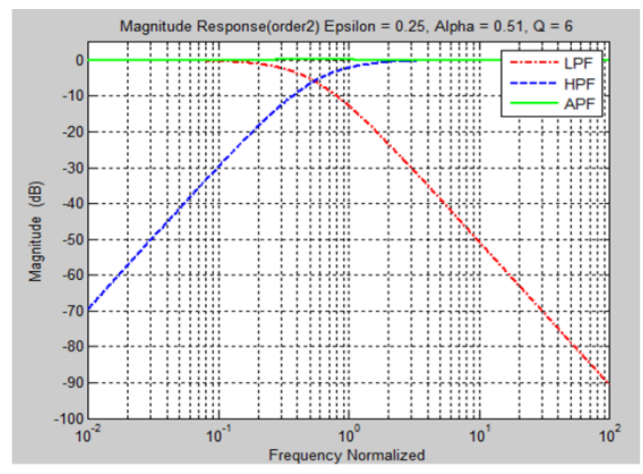

(a)

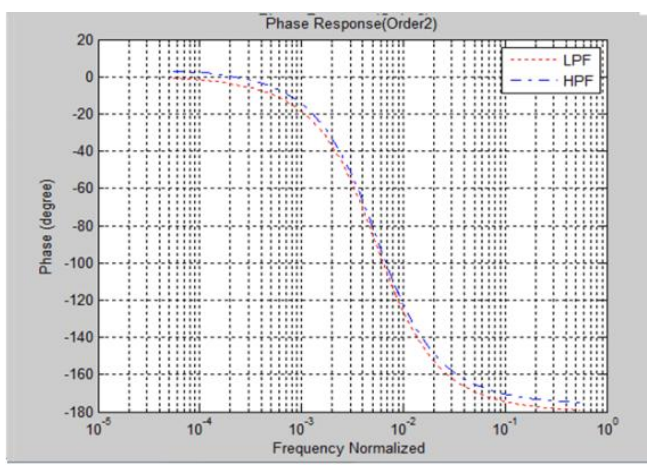

(b)

Fig. 4: Frequency responses of the proposed two-band crossover network. (a) Magnitude of LPF, HPF and their summed and (b) phase response. 


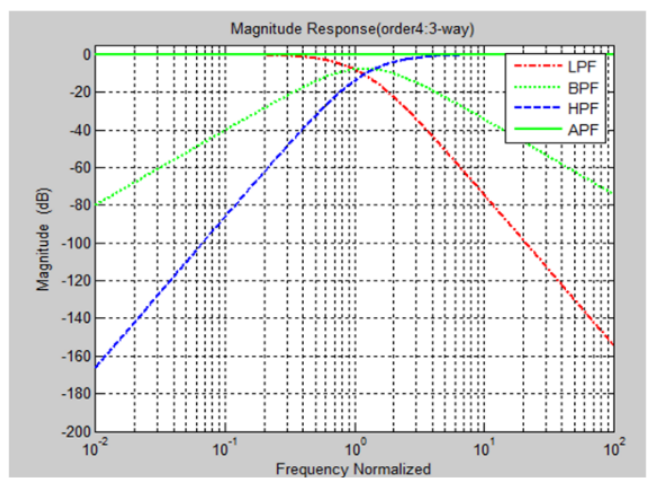

(a)

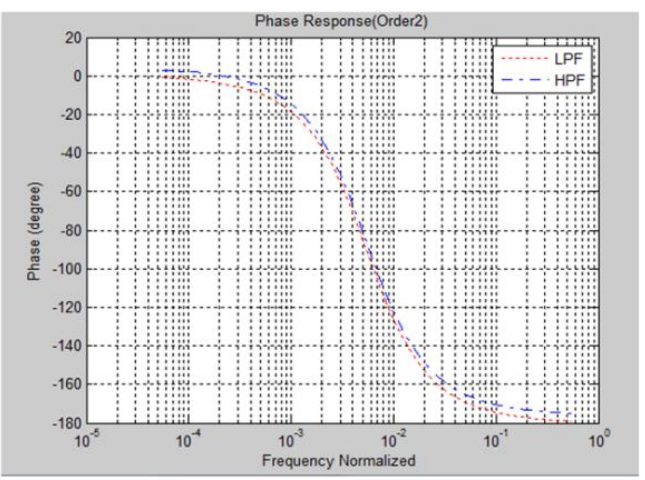

(b)

Fig. 5: Frequency responses of the proposed three-band crossover network. (a) magnitude responses and their summed and (b) phase Responses.

From Fig. 4 shows the frequency response of the proposed two-band crossover networks. The comparison of the magnitude responses is shown in Fig. 4a. It shown that the slope of both LPF and HPF is about $10 \mathrm{~dB} /$ Octave, which is higher than the Butterworth filter at the same second-order. The summed of the magnitude responses is flat $(0 \mathrm{~dB}$. $)$ whole all frequencies. The phase response of the LPF and the HPF are same the APF. The frequency responses of the three-way crossover network are depicted in Fig. 5. The slope of the LPF and HPF is high rate and their summed magnitude is also flat at all frequencies. The phase responses of all filters are rather same phase which meet the in-phase properties.

\section{Conclusion}

The new two and three ways crossover network based on q-Bernstein filters having in-phase and flat summed of magnitude response properties with varying slope rates by changing variable parameters of the filter coefficients is presented. The proposed crossover network is less of complexness due to a useful of subtractive structure form and provided a high slope rate in transition band more than 10 and $20 \mathrm{~dB} /$ octave for the second-order and fourth-order of LPF, respectively.

\section{Acknowledgements}

This work was supported by KMITL-UEC Global Alliance Lab Project 2015. We would like to express my sincere thanks to Prof. Dr. Hisayuki AOYAMA. Furthermore, we also sincerely thank Associate Professor Dr. Kanok Janchitrapongvej for his recommended.

\section{Reference}

[1] S. P. Lipshitz, J. Vanderkooy. A Family of Linear-Phase Crossover Network of High Slope Derived by Time Delay. JAES. 1983, vol. 31, pp. 2 - 20.

[2] J. Vanderkooy, S. P. Lipshitz. In Phase Crossover Design. JAES. 1986. vol. 34, pp. 889 - 894.

[3] J. A. D' Appolito. Active Realization of Multiway All-Pass Crossover Systems. JAES. (Abstracts). 1984. vol. 32, p. 1008.

[4] P. Garde. All-Pass Crossover Systems. JAES. 1980. vol. 28, pp. 575 - 584.

[5] S. H. Linkwitz. Active Crossover Networks for Noncoincident Drivers. JAES. 1976. vol. 24, pp. 2 - 8.

[6] V. Chutchavong. Linear-Phase Bernstein Filters for Equalized and the Distorted Chrominance. LAP LAMBERT A CADEMIC Publishing. Germany. 2013.

[7] V. Kuc, P. Cheung. Quantum Calculus. 2000. Springer.

[8] R. Christensen. Active All-Pass Crossover Network with Equal Resistors and Equal Capacitors. JAES. 2006. Vol. 54. No. $1 / 2$, pp.45-53. 